\title{
Substituição da aorta ascendente e arco aórtico por enxerto de pericárdio bovino: resultados a médio prazo
}

Fernando Antônio FANTINI*, Bayard GONTIJO FILHO*, Mário O. VRANDECIC*, Juscelino Teixeira BARBOSA $^{*}$, João Alfredo PAULA E SILVA*, Leonardo F. DRUMOND*, Eduardo Peredo ALCOCER ${ }^{*}$, Marcelo F. CASTRO*, Arturo B. FERRUFINO*, Flávio J. MACIEL*, Maurício R. BARBOSA*, Maria A. BRAGA*.

FANTINI, F. A.; GONTIJO FILHO, B.; VRANDECIC, M. O.; BARBOSA, J. T.; PAULA E SILVA, J. A.; DRUMOND, L. F.; ALCOCER, E. P.; CASTRO, M. F.; FERRUFINO, A. B.; MACIEL, F. J.; BARBOSA, M. R.; BRAGA, M. A. - Substituição da aorta ascendente e arco aórtico por enxerto de pericárdio bovino: resultados a médio prazo. Rev. Bras. Cir. Cardiovasc., 9 (2): 81-87, 1994.

RESUMO: Condutos e remendos de pericárdio bovino (PB) têm sido utilizados na substituiçāo ou reparo da aorta ascendente e segmentos iniciais do arco aórtico, na nossa Instituição desde 1989. As principais vantagens são a facilidade de seu manuseio, a boa hemostasia, o baixo custo. Este estudo foi realizado para analisar o comportamento desse heteroenxerto após um mínimo de 2 anos de implante. No período de fevereiro de 1989 a fevereiro de 1994.83 pacientes foram submetidos a 85 procedimentos com implante de enxertos de PB na aorta ascendente ou arco aórtico. A mortalidade hospitalar foi de $18 \%$. Trinta e um pacientes com pelo menos 2 anos de seguimento foram selecionados para análise do enxerto (seguimento médio $=40,9$ meses). Foram submetidos a estudo clínico e ecocardiográfico, sendo 15 reestudados através de cineangiocardiografia. Foram pesquisados o desempenho hemodinâmico e a presença de alteraçōes, tais como dilatação, calcificação, trombose ou pseudoaneurisma. Em todos os pacientes o enxerto estava funcionando perfeitamente e sem ocorrência de qualquer anormalidade. O presente estudo permite concluir que, em até 5 anos, não se observou qualquer alteraçāo adversa no funcionamento desses enxertos, que se mostraram de mais fácil manuseio e mais hemostáticos que os enxertos de Dacron classicamente usados.

DESCRITORES: aorta ascendente, cirurgia; arco aórtico, cirúrgia; enxertos pericárdicos.

\section{INTRODUÇÃO}

As propriedades hemostáticas, a facilidade de manuseio e o baixo custo do enxerto de pericárdio bovino na correção de lesões da aorta $1,11,12$ tornam esse tipo de substituto vascular uma opção técnica bastante atraente. No entanto, dúvidas quanto a seu desempenho tardio persistem, especialmente com relação a possibilidade de dilatação, calcificação, formação de trombos ou desenvolvimento de falso aneurisma nas linhas de sutura.
O uso do enxerto de pericárdio bovino tratado por processo específico à base de glutaraldeído (e produzido por Biocor Indústria e Pesquisas, Belo Horizonte - MG) no tratamento de aneurismas da aorta ascendente é rotina na nossa Instituição, desde 1989, como conduto simples ou valvulado e sob a forma de remendo. Com o objetivo de estudar o comportamento desses heteroenxertos a médio prazo, apresentamos a evolução de um grupo de pacientes com pelo menos 2 anos de acompanhamento.

Trabalho realizado no Biocor Instituto, Belo Horizonte, MG, Brasil.

Apresentado do $21^{\circ}$ Congresso Nacional de Cirurgia Cardiaca. Porto Alegre, RS, 24 e 25 de março, 1994.

- Do Biocor Hospital de Doenças Cardiovasculares, Belo Horizonte, MG.

Endereço para correspondência: Fernando A. Fantini. Caixa Postal 106. CEP 30161-970, Belo Horizonte, MG, Brasil. 
FANTINI, F. A.; GONTIJO FILHO, B.; VRANDECIC, M. O.; BARBOSA, J. T.; PAULA E SILVA, J. A.; DRUMOND, L. F.; ALCOCER, E. P.; CASTRO, M. F.; FERRUFINO, A. B.; MACIEL, F. J.; BARBOSA, M. R.; BRAGA, M. A. - Substituiçāo da aorta ascendente e arco aórtico por enxerto de pericárdio bovino: resultados a médio prazo. Rev. Bras. Cir. Cardiovasc., 9 (2): 81-87, 1994.

\section{CASUÍSTICA E MÉTODOS}

No período compreendido entre abril de 1986 e fevereiro de 1994, 240 pacientes portadores de aneurisma de diversos segmentos da aorta foram submetidos a 242 procedimentos cirúrgicos (Gráfico 1). A mortalidade hospitalar geral foi de $21 \%$ ( 51 pacientes). Desse grupo, 96 pacientes apresentavam acometimento da aorta ascendente, sendo que em 85 operações foram utilizados heteroenxertos de pericárdio bovino na correção cirúrgica, como descrito a seguir: 33 condutos valvulados, 47 condutos não valvulados e 5 remendos simples (Gráfico 2). Nesse subgrupo a mortalidade hospitalar foi de $18 \%$ (15 pacientes).

Trinta e um dentre 33 pacientes operados de fevereiro de 1989 a fevereiro de 1991 estão sendo acompanhados, num período variando de 2 a 5 anos (média: 40,9 meses), com idades entre 27 e 77 anos (média: 50,6 anos), sendo 21 do sexo masculino e 10 do sexo feminino. A doença primária $e$ as condições associadas estão sumarizadas nas Tabelas 1 e 2 . As dissecções agudas e crônicas

\section{GRÁFICO 1}

PROCEDIMENTOS CIRÚRGICOS REALIZADOS EM 240 PACIENTES PORTADORES DE LESĀO AORTICA DE ACORDO COM A TOPOGRAFIA.

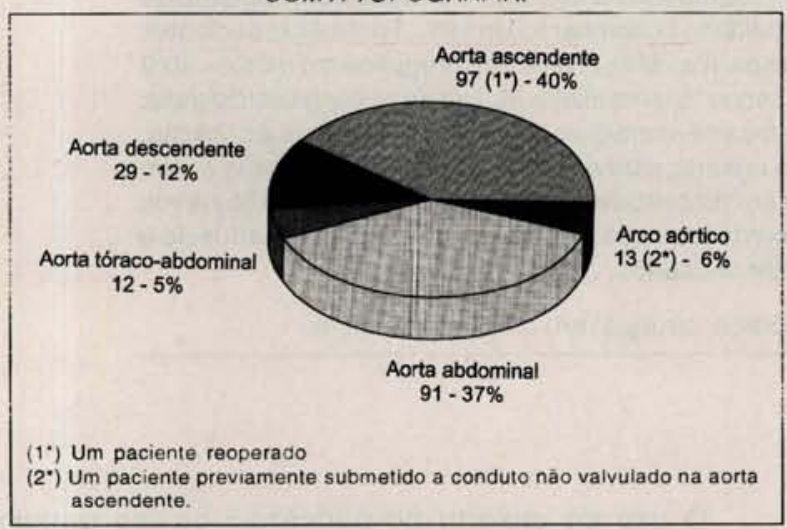

GRÁFICO 2

TIPOS DE ENXERTOS DE PERICÁRDIO BOVINO UTILIZADOS EM 85 PROCEDIMENTOS CIRÚRGICOS DE 1989 A 1994

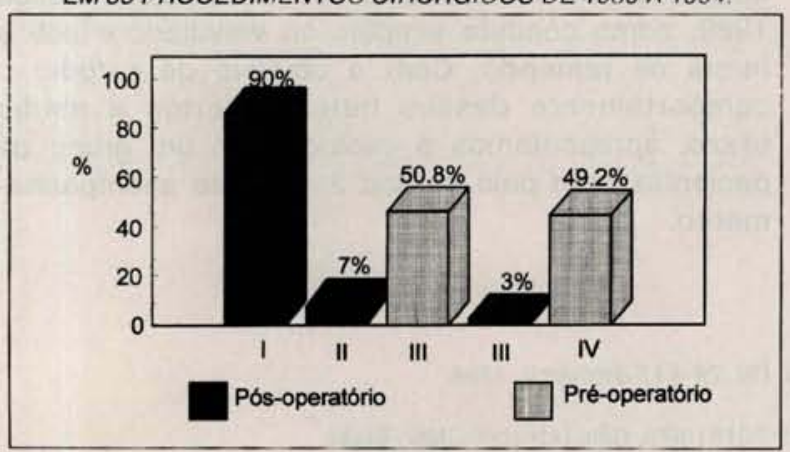

TABELA 1

LESĀO AÓRTICA PRIMÁRIA DE 31 PACIENTES SUBMETIDOS A CORRECAÃO CIRÚRGICA E COM ACOMPANHAMENTO SUPERIOR A 2 ANOS

\begin{tabular}{lrr}
\hline LESÃO & N. & $\%$ \\
\hline Dissecção aguda da aorta tipo A & 16 & 51,6 \\
Dissecção crônica da aorta tipo A & 5 & 16,1 \\
Aneurisma fusisforme da aorta ascendente & 7 & 22,6 \\
Aneurisma sacular do arco aórtico & 2 & 6,5 \\
Aneurisma sacular da aorta ascendente & 1 & 3,2 \\
\hline Total & 31 & 100,0 \\
\hline
\end{tabular}

TABELA 2

LESĀO ASSOCIADA PRESENTE EM 31 PACIENTES SUBMETIDOS A CORREÇĀO DE DOENÇAS DA AORTA ASCENDENTE

\begin{tabular}{lrr}
\hline LESĀO & N. & $\%$ \\
\hline Coronariopatia & 6 & 19,4 \\
Insuficiência mitral severa & 1 & 3,2 \\
Compressāo extrínseca de art. pulmonar & 1 & 3,2 \\
Síndrome de compressão da VCS & 1 & 3,2 \\
Fenda de dissecção no arco aórtico & 2 & 6,5 \\
\hline Total & 11 & 35,5 \\
\hline
\end{tabular}

VCS $=$ Veia cava superior

envolvendo a aorta ascendente foram as afecçōes mais freqüentes.

Dentre os aneurismas fusiformes, 2 pacientes tinham síndrome de Marfan e 1 estenose aórtica severa, 2 eram arterioscleróticos e 1 sifilítico. Quanto à etiologia dos aneurismas saculares, 2 eram micóticos e em 1, acometendo a aorta ascendente, não foi possível determinar o agente causal.

Coronariopatia significativa foi detectada em $20 \%$ dos casos. Em 2 pacientes os sinais e sintomas de compressāo extrínseca foram os responsáveis pelo diagnóstico. Um paciente com síndrome de Marfan apresentava insuficiência mitral severa e 2, fendas de reentrada no arco aórtico.

\section{TÉCNICA CIRÚRGICA}

Esternotomia mediana foi a incisão empregada em todos os pacientes. Após estabelecimento da circulação extracorpórea, 23 pacientes foram submetidos a hipotermia moderada $\left(26^{\circ} \mathrm{C}\right)$ e 8 a hipotermia profunda $\left(18^{\circ} \mathrm{C}\right) \mathrm{com}$ parada cardiocirculatória total. Ultimamente temos empregado a parada cardiocirculatória e a técnica de open distal anastomosis, como preconizado por COOLEY \& LIVESAY 6 em quase todos os casos de dissecçäo de aorta, per- 
FANTINI, F. A.; GONTIJO FILHO, B.; VRANDECIC, M. O.; BARBOSA, J. T.; PAULA E SILVA, J. A.; DRUMOND, L. F.; ALCOCER, E.P.; CASTRO, M. F.; FERRUFINO, A. B.; MACIEL, F.J.; BARBOSA, M. R.; BRAGA, M. A. - Substituição da aorta ascendente e arco aórtico por enxerto de pericárdio bovino: resultados a médio prazo. Rev. Bras. Cir. Cardiovasc., 9 (2): 81-87, 1994.

mitindo, assim, a inspeção do arco aórtico e maior facilidade na feitura da anastomose distal. Assim, a presença de fendas no arco aórtico em 2 dos casos acompanhados foi disgnosticada e tratada no mesmo ato cirúrgico. Para a proteção miocárdica foi utilizada a cardioplegia cristalóide nos óstios coronários.

Os enxertos são de pericárdio bovino tratado com glutaraldeido. Os condutos simples são suturados apenas no seu terço médio, de modo que as extremidades abertas facilitam o manuseio do material. Os condutos valvulados contêm válvula biológica standart implantada em uma de suas extremidades e também são apresentados com a extremidade distal aberta.

Basicamente, cinco foram as técnicas cirúrgicas empregadas (Tabela3).

TABELA 3

TÉCNICA CIRÜRGICA UTILIZADA EM 31 PACIENTES SUBMETIDOS A CORRECÃO DE LESÕES DA AORTA ASCENDENTE

\begin{tabular}{lrr}
\hline OPERAÇĀO & $N$ & $\%$ \\
\hline Conduto não valvulado & 14 & 45,1 \\
Conduto não valvulado + Troca valva aórtica & 4 & 12,9 \\
Bentall e De Bonno clássico & 7 & 22,6 \\
Conduto valvulado + reimplante coronária & 3 & 9,7 \\
Remendo simples & 3 & 9,7 \\
\hline Total & 31 & 100,0 \\
\hline
\end{tabular}

1) Implante de conduto não valvulado, até 1990 , quando era usada cola biológica de gelatinaresorcina-formol e tiras de pericárdio bovino para reconstituição da parede aórtica proximal e distal, nos casos de dissecção da aorta e o tubo de pericárdio suturado proximal e distalmente com chuleio de fio do tipo Poliester trançado 3-
0. A partir de então, passou-se a ressecar todo o tecido doente e se abandonou o uso da cola, sendo que a boca proximal do tubo talhada para acompanhar o perfil da aorta ressecada. As suturas passaram a ser feitas com fio de Polipropilene 4-0.

2) Implante de conduto não valvulado + troca de valva aórtica: após ressecção das válvulas; a prótese biológica Biocor era implantada com pontos separados em "U" e a interposição do conduto feita da mesma forma do ítem 1.

3) Bentall e De Bono clássico: após incisão longitudinal do aneurisma e ressecção das válvulas aórticas, o tubo de pericárdio valvulado foi suturado ao anel aórtico com pontos em "U" supra-anulares de fios de Poliester trançado 20 . As anastomoses dos óstios coronários ao conduto, assim como a anastomose distal, foram feitas com fio de Polipropilene 4-0. A capa do aneurisma foi usada para cobertura do enxerto, após revisão cuidadosa da hemostasia.

4) Conduto valvulado + reimplante de coronárias: variante da técnica de Bentall e De Bono em que um ou ambos os óstios coronários foram destacados da parede da aorta sob a forma de botão e suturados diretamente no tubo com chuleio de Polipropilene 4-0; toda a parede aórtica foi ressecada.

5) Remendo simples: nos aneurismas saculares, remendo de pericárdio bovino foi suturado na boca do aneurisma com chuleio de Polipropilene 4-0.

As técnicas cirúrgicas utilizadas estão relacionadas na Tabela 4

Em cerca de $25 \%$ dos casos, outros procedimentos foram realizados (Tabela 5 ). A revascularização do miocárdio foi necessária em 5 pacientes, sendo que em bypass aorto-tronco braquiocefálico foi associado. As fendas no arco aórtico foram

TABELA 4

TÉCNICAS CIRÚRGICAS UTILIZADAS EM 31 PACIENTES DE ACORDO COM A LESÃO PRIMÁRIA DA AORTA

\begin{tabular}{|c|c|c|c|c|c|}
\hline & DIS. AGUDA & DIS. CROANICA & $\begin{array}{c}\text { LESÃO DA AORTA } \\
\text { AN. FUS. }\end{array}$ & $A N$. SAC. & TOTAL \\
\hline Cond. $n$ / valvulado & 11 & 3 & - & - & 14 \\
\hline Cond. $n$ / valvulado + TVA & 1 & 1 & 2 & - & 4 \\
\hline Cond. valvulado + reimpl. cor. & 3 & - & - & - & 3 \\
\hline Bentall clássico & - & 2 & 5 & - & 7 \\
\hline Remendo simples & - & - & - & 3 & 3 \\
\hline Total & 15 & 6 & 7 & 3 & 31 \\
\hline
\end{tabular}

Cond.$=$ Conduto, Valv. $=$ Valvulado, Reimp. Cor. $=$ Reimplante de Coronária 
FANTINI, F. A.; GONTIJO FILHO, B.; VRANDECIC, M. O.; BARBOSA, J. T.; PAULA E SILVA, J. A.; DRUMOND, L. F.; ALCOCER, E.P.; CASTRO, M. F.; FERRUFINO, A. B.; MACIEL, F.J.; BARBOSA, M. R.; BRAGA. M. A. - Substituição da aorta ascendente e arco aórtico por enxerto de pericárdio bovino: resultados a médio prazo. Rev. Bras. Cir. Cardiovasc., 9 (2): 81-87, 1994.

TABELA 5

OPERACOOEES ASSOCIADAS EM 31 PACIENTES SUBMETIDOS A CORREÇĀO DE LESÖES DA AORTA ASCENDENTE

\begin{tabular}{lcc}
\hline OPERAÇÃO & $N$. & $\%$ \\
\hline Revascularização do miocárdico & 3 & 9,7 \\
Revascularização do miocárdico & 2 & 6,5 \\
$\quad$ By-pass Ao-TBC & 1 & 3,2 \\
Troca de valva mitral & 1 & 3,2 \\
Tromba de elefante aorta descendente & 1 & 3,2 \\
Sutura de fenda no aorco aórtico & 8 & 25,8 \\
\hline Total
\end{tabular}

Ao-TBC = aorta-tronco braquiocefálico

corrigidas através de sutura direta em 1 caso e pela técnica de "tromba de elefante" 5 em outro. Um paciente, portador de síndrome de Marfan, foi submetido a troca da valva mitral.

\section{SEGUIMENTO}

Os pacientes em acompanhamento foram submetidos a avaliação clínica periódica e exames complementares, como exame radiológico do torax, ecocardiograma convencional ou transesofágico e tomografia computadorizada. Quinze deles foram reestudados através de cineangiocardiografia em período que variou de 6 a 52 meses (Figuras 1 e 2). Foram pesquisados o desempenho hemodinâmico, a presença de dilatação, calcificação ou trombose ao nivel do enxerto e a formação de pseudoaneurisma nas anastomoses. Em 2 pacientes reoperados foi possível a análise direta das próteses.

\section{RESULTADOS}

Excluindo-se $15(18 \%)$ pacientes que foram a óbito no período hospitalar e 2 outros em que não foi possivel obter informações sobre a evolução pós-

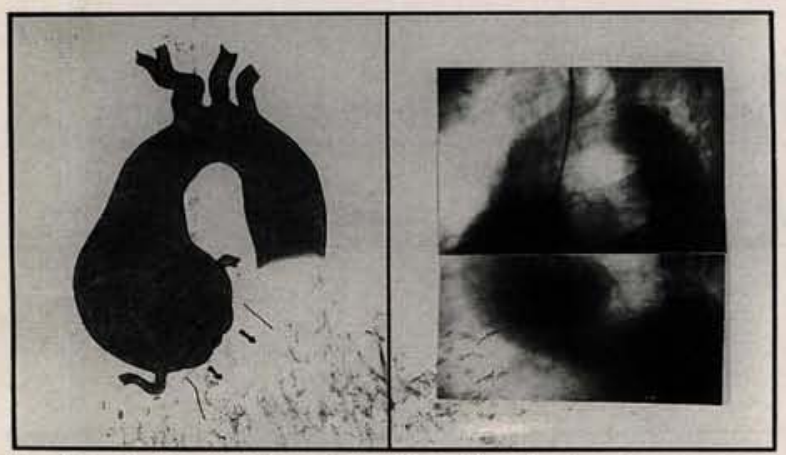

Fig. 1 - Esquema e cineangiocardiografia pré-operatória de um dos pacientes estudados mostrando aneurisma fusiforme da aorta ascendente com insuficiência aórtica importante.

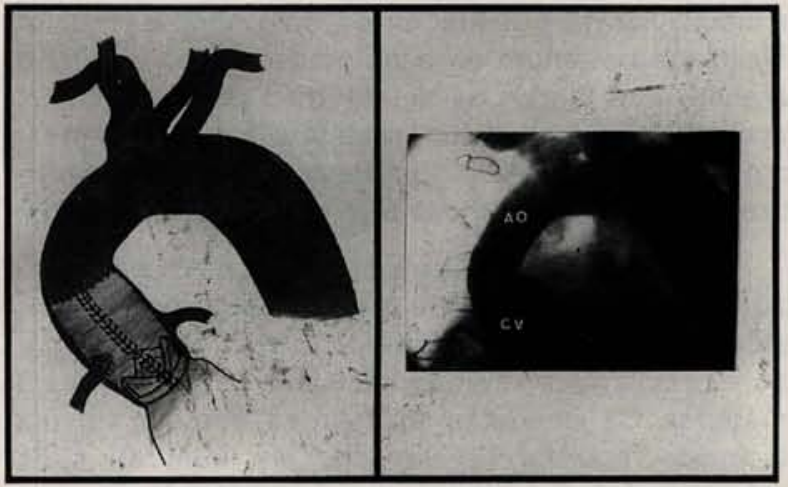

Fig. 2 - Cirurgia de Bentall e de Bono realizada no mesmo paciente, com o respectivo reestudo pós-operatório mostrando função adequada do enxerto.

operatória, 31 pacientes foram seguidos por um período que variou de 24 a 60 meses (média 40,9 meses).

Ocorreram 2 óbitos tardios, um deles 35 meses após a operação, devido a câncer de próstata e o outro no $38^{\circ}$ mês de pós-operatório, devido a infarto agudo do miocárdio, em paciente de 77 anos submetida a correção de aneurisma sacular da aorta ascendente com compressão da veia cava superior.

Dois pacientes foram reoperados aos 34 e 56 meses de pós-operatório, em decorrência de alteraçōes relacionadas à doença de base e sem relação direta com os enxertos. Ambos, portadores de dissecção aguda da aorta, haviam sido submetidos a implante de tubos não valvulados e uso de cola biológica na aorta ascendente. No seguimento pósoperatório evoluíram com dilataçāo do início da aorta ascendente e insuficiência aórtica. O primeiro paciente, que apresentava permanência da fenda de dissecção no arco aórtico, foi submetido a substituição do tubo inicial por conduto valvulado de pericárdio bovino com reimplante das artérias coronárias e substituição do arco aórtico por tubo de Dacron de baixa porosidade. No segundo caso, foi empregada a técnica de Bentall e De Bono para implante de um conduto valvulado de pericárdio bovino e revascularização do miocárdio concomitante. Durante as operações foi observado o perfeito estado dos enxertos, que apresentavam sinais discretos de degeneração sem calcificação.

Os demais pacientes encontram-se em classe funcional I ou II. Não há evidências de dilatação, calcificação, trombose, formação de pseudoaneurisma ou de disfunção das biopróteses.

\section{COMENTÁRIOS}

O sangramento continua sendo uma das principais causas de morbidade e mortalidade no trata- 
FANTINI, F. A.; GONTIJO FILHO, B.; VRANDECIC, M. O.; BARBOSA, J. T.; PAULA E SILVA. J. A.; DRUMOND, L. F.; ALCOCER, E. P.; CASTRO, M.F.; FERRUFINO, A. B.; MACIEL, F.J.; BARBOSA, M. R.; BRAGA, M. A. - Substituição da aorta ascendente e arco aórtico por enxerto de pericárdio bovino: resultados a médio prazo. Rev. Bras. Cir. Cardiovasc., 9 (2): 81-87, 1994.

mento cirúrgico da aorta torácica $10,20,24$. Considerando-se que a circulação extracorpórea hipotérmica por si só já acarreta distúrbios de coagulação ${ }^{15}, 11$, as táticas cirúrgicas têm sido fortemente influenciadas pela natureza porosa das próteses de Dacron classicamente empregadas. Diversas técnicas de pré-coagulação ${ }^{4,7}$, o uso de próteses impregnadas por colágeno ${ }^{27}$, asssim como o emprego da capa aneurismática para cobertura dos enxertos ${ }^{2}$, têm sido utilizados para minimizar este grave problema. Nesse aspecto, o pericárdio bovino apresenta vantagens com relação aos tubos de Dacron, já que, por não serem porosos, são mais hemostáticos 1,11 e, além disso, de mais fácil manuseio.

A formaçāo de pseudoaneurisma nas linhas de sutura 17,25 e a incidência maior de endocardite 13,16 relacionada ao uso de tubos de Dacron ou à técnica cirúrgica têm sido relatadas na literatura. A evolução a longo prazo dos condutos de pericárdio bovino é, no entanto, pouco conhecida e divulgada, e alterações como dilatação, degeneração e calcificação são passíveis de ocorrer.

A formação de pseudoaneurismas nas anastomoses coronárias foi primariamente descrita por KOUCHOUKOS et alii ${ }^{17}$. Segundo os autores o hematoma de alta pressão que se forma entre o enxerto e a capa aneurismática seria a causa da complicação. No entanto, relatos de formação de pseudoaneurismas de casos em que o revestimento não foi utilizado têm sido publicados ${ }^{13}$. MERRILL et alii ${ }^{19}$, após estudos em 2 pacientes que desenvolveram a complicação aos 13 e 23 anos de pósoperatório, concluíram que uma cicatrização verdadeira entre a prótese e a artéria nunca ocorre; portanto, a integridade da anastomose dependeria da manutenção da tensão da sutura durante a vida do paciente 22 . Nos nossos pacientes esse tipo de problema não foi observado até o momento. Acreditamos que a maleabilidade do enxerto, que proporcionaria uma melhor coaptação das bordas suturadas e a formação de tecido fibroso na face interna do enxerto observada em estudos experimentais por GABBAY et alii ${ }^{9}$, sejam fatores determinantes de um melhor prognóstico neste aspecto. Assim, continuamos a efetuar o fechamento da capa aneurismática sobre o conduto, conforme originalmente proposto por BENTALL \& DE BONO 2

Diversos autores têm divulgado os problemas de degeneração e calcificação que ocorrem com as próteses valvulares de pericárdio bovino, especialmente em pacientes jovens 23 . Estudos recentes sugerem que o glutaraldeído pode induzir resposta imunológica in vivo, consistente com uma reação do tipo hospedeiro-versus-prótese ${ }^{8}$. GABBAY et alii 9 realizaram estudo experimental em cães, implan- tando pericárdio bovino tratado com glutaraldeído em diferentes locais do coração e observaram que, em contato com sangue e endocárdio (parede atrial esquerda), o pericárdio ficava espessado e se calcificava rapidamente e como substituto de cordas tendineas, apesar da calcificação, mantinha as suas dimensões originais. Além disso, não havia formação de neoíntima, mas a face interna do enxerto era recoberta por tecido fibroso a partir das suturas.

Essas observações são de fundamental importância, pois comprovam a resistência do pericárdio ao estresse físico, na forma de pressão ou tensão e corroboram com os resultados que temos obtido com esse material. Não há evidências de rotura ou dilatação dos enxertos implantados em nenhum dos pacientes operados. Nos dois reoperados o pericárdio encontrava-se mais espessado e com fibrose externa e interna ao nivel das suturas, porém sem qualquer evidência das complicações citadas. A calcificação dos tecidos que provavelmente ocorrerá somente preocupa quanto às válvulas biológicas dos tubos valvulados, já que a calcificação das paredes do conduto certamente não irá comprometer a função tubular.

Estudos experimentais e clínicos em andamento na nossa Instituição, com um novo método de tratamento anticalcificante* 26 , têm produzido tecidos biológicos $100 \%$ citocompatíveis, não acarretando, portanto, reação de corpo estranho. Não ocorreu calcificação nos modelos animais e o material mostrou uma resistência tênsil cinco vezes maior que a obtida com os tratamentos convencionais. A superfície dos enxertos, além do mais, permite a proliferação andotelial e, assim, a formação de neointima. Acreditamos que este tratamento, totalmente histocompativel, deva determinar maior longevidade ao heteroenxerto, melhorando, assim, os resultados.

Quanto à técnica cirúrgica, nos casos de dissecção da aorta, os 2 casos reoperados nos mostraram que é essencial a ressecção proximal de todo o tecido doente. Não temos, portanto, feito uso da cola biológica ${ }^{14}$, como alguns grupos nacionais 3,21 , já que a sutura proximal é feita em tecido sadio e com fios delicados, e o enxerto pode ser moldado para acompanhar o perfil da boca anastomótica. Quanto à sutura distal, temos utilizado, de rotina, a técnica de open distal anastomosis como preconizada por COOLEY \& LIVESAY ${ }^{6}$, pois consideramos essencial a inspeção do arco aórtico. LANSMAN et alii ${ }^{18}$, em recente artigo, descrevem uma incidência de fendas no arco aórtico de $31 \%$.

Desde que problemas tardios, como calcificação

-No-React ${ }^{\mathrm{TM}}$ 
FANTINI, F. A.; GONTIJO FILHO, B.; VRANDECIC, M. O.; BARBOSA, J. T.; PAULA E SILVA, J. A.; DRUMOND, L. F.; ALCOCER, E. P.; CASTRO, M. F.; FERRUFINO, A. B.; MACIEL, F.J.; BARBOSA, M. R.; BRAGA. M. A. - Substituição da aorta ascendente e arco aórtico por enxerto de pericárdio bovino: resultados a médio prazo. Rev. Bras. Cir. Cardiovasc., 9 (2): 81-87, 1994.

e formação de pseudoaneurisma, podem ocorrer, é necessário um período de seguimento mais longo. Entretanto, tendo-se em vista a excelente evolução dos pacientes operados e a ausência de complicações relacionadas à protese em até cinco anos de observação, podemos concluir que os enxertos de pericárdio bovino, quando usados na aorta ascendente e arco aórtico, são excelentes substitutos vasculares e se mostram de fácil manuseio, hemostáticos e de baixo custo.

RBCCV 44205-234

FANTINI, F. A.; GONTIJO FILHO, B.; VRANDECIC, M. O.; BARBOSA, J. T.; PAULA E SILVA, J. A.; DRUMOND, L. F.; ALCOCER, E. P.; CASTRO, M. F.; FERRUFINO, A. B.; MACIEL, F. J.; BARBOSA, M. R.; BRAGA, M. A. - Replacement of the ascending aorta and aortic arch by bovine pericardial grafts. Rev. Bras. Cir. Cardiovasc., 9 (2): 81-87, 1994.

ABSTRACT: Bovine pericardial (P.B.) grafts are in clinical use at Biocor Institute since 1989 for replacement or repair of the ascending aorta ( $A S A O$ ) and the initial segment of the aortic arch ( $A \circ A r$ ); the main advantages of B.F. grafts that justified its clinical application are: easiness to handle, fully hemostatic and low cost. This study was conducted to analyse the fate of the heterograft after a minimum of 2 years of implantation. From February 1989 to Frebruary 1994, 84 patients had a B.P. graft implanted in the AsAo or AoAr. The total hospital mortality was $18 \%$. Thirty-one patients who had two years of follow-up were selected for analysis of the B.P. graft (mean follow-up $=40.9$ months). They underwent either an echocardiographic examination or an aortographic study $(n=15)$ in order to determine the presence of dilatation, calcification, thrombus and pseudoaneurysm. In all patients the graft was functioning perfectly and no abnormalities were recorded. The current follow-up is short and complications such as calcification and pseudoaneurysm may still develop. therefore a longer time is required. The present study has shown superior handling and hemostatic properties over the classical Dacron grafts and no adverse behavior was noted during this 4 years of study.

DESCRIPTORS: ascending aorta, surgery; aortic arch, surgery; pericardial grafts, surgery.

\section{REFERÊNCIAS BIBLIOGRÁFICAS}

1 ARDITO, R. V.; SANTOS, J L. V.; MAYORQUIM, R. C.; GRECO, O. T.; ZAIATCHICK, M.; SOTO, H. G.; JACOB, J. L. B.; BRAILE, D. M. - Substituição completa da aorta ascendente e da valva aórtica com tubo valvulado de pericárdio bovino. Rev. Bras. Cir. Cardiovasc., 2: 129-138, 1987.

BENTALL, H. H. \& DE BONO, A. - A technique for complete replacement of ascending aorta. Thorax, 23: 338-339, 1968.

BERLINCK, M. F.; BRITO, J. O. R.; ROJAS, S. S. O.; SOUZA, J. M.; OLIVEIRA, S. A. - Tratamento cirúrgico da dissecçāo da aorta. Rev. Bras. Cir. Cardiovasc., 5: 61-65, 1990.

BETHEA, M. C. \& REEMTSMA, K. - Graft hemostasis: an alternative to pre-clotting. Ann. Thorac. Surg., 27: $374,1979$.

BORST, H. G.; WALTERBUSCH, G.; SCHAPS, D. Extensive aortic replacement using "elephant trunk" prosthesis. Thorac. Cardiovasc. Surg., 31: 37-40, 1983.
6 COOLEY, D. A. \& LIVESAY, J. J. - Technique of open distal anastomosis for ascending and transverse aortic resection. Cardiovasc. Dis., 8: 421-426, 1981.

7 COOLEY, D. A.; ROMAGNOLI, A.; MILAN, J. D.; BOSSANT, M. L. - A method for preparing woven Dacron grafts to prevent intersticial hemorrhage. Bull. Texas Heart Inst., 8: 48, 1991.

8 DAHM, M.; LYMAN, W. D.; SCHWELL, A. B.; FRATER, R. W. M. - Immunogenicity of glutaraldehydetanned bovine pericardium. J. Thorac. Cardiovasc. Surg., 99: 1082-1090, 1990.

9 GABBAY, S.; BORTOLOTTI, U.; FACTOR, S.; SHORE, D. F.; FRATER, M. B. - Calcification of implanted xenograft pericardium. Influence of site and function. J. Thorac. Cardiovasc. Surg., 87: 782-787, 1984.

10 GARCIA-MACEDO, R.; KALIL, R. A. K. M.; PRATES, P. R.; LUCCHESE, F. A.; SANT'ANA, J. R.; PEREIRA, E. M.; COSTA, A. R.: LARA, F. R.; NESRALLA, I. A. - Tratamento cirúrgico para ectasia ânulo-aórtica. Rev. Bras. Cir. Cardiovascular., 1: 44-48, 1986.

11 GONTIJO FILHO, B.; FANTINI, F. A.; BARBOSA, J. T.; 
FANTINI, F. A.; GONTIJO FILHO, B.; VRANDECIC, M. O.; BARBOSA, J. T.; PAULA E SILVA, J. A.; DRUMOND. L. F.; ALCOCER, E. P.; CASTRO, M. F.; FERRUFINO, A. B.; MACIEL, F.J.; BARBOSA, M. R.; BRAGA, M. A. - Substituição da aorta ascendente e arco aórtico por enxerto de pericárdio bovino: resultados a médio prazo. Rev. Bras. Cir. Cardiovasc., 9 (2): 81-87, 1994.

SILVA, J. A. P.; CASTRO, M. F.; PEREDO, E. O. A.; PEDROSA, A. A.; GONÇALVES, F. D.; VRANDECIC, M. O. - Correção de doenças da aorta torácica com utilização de hipotermia profunda e parada circulatória. Rev. Bras. Cir. Cardiovasc., 6:11-16, 1991.

GONTIJO FILHO, B.; FANTINI, F. A.; SILVA, J. A. P.; BARBOSA, J. T.; MASCI, M. G. J.; FREIRE, R. J. A.; SOUZA, R. P. - Correçāo cirúrgica da estenose supra-aórtica localizada; relato de 2 casos. Arq. Bras. Cardiol., 43: 433-436, 1984.

13 GOTT, V. L.; PYERITZ, R. E.; CAMERON, D. E.; GREENE, P. S.; MCKUSICK, V. A. - Composite graft repair of Marfan aneurysm of the ascending aorta: results in 100 patients. Ann. Thorac. Surg., 52: 3845, 1991.

14 GUILMET, D.; BACHET, J.; GOUDOT, B.; LAURIAN, C.; GIGOU, F.; BICAL, O.; BARBAGELLATA, M. Use of biological glue in acute aortic dissection: preliminary clinical results with a new surgical technique. J. Thorac. Cardiovasc. Surg., 77: 516$521,1979$.

HARKER, L. A. - Bleeding after cardiopulmonary by pass. N. Engl. J. Med., 314: 1446-1448, 1986.

16 KALIL, R. A. K.; GARCIA-MACEDO, R.; PRATES, P. R.; LUCCHESE, F.; SANT'ANNA, J. R.; LARA, R. F. A.; COSTA, A. R.; DAUDT, N. S.; PEREIRA, E. M.; NESRALLA, I. A. - Reoperações após cirurgia de Bentall-de Bono para ectasia ânulo-aórtica. Rev. Bras. Cir. Cardiovasc., 3: 93-100, 1988.

17 KOUCHOUKOS, N. T.; MARSHALL, W. C.; WEDIGESTECHER, T.A. - Eleven-year experience with composite graft replacement of the ascending aorta and aotic valve. J Thorac. Cardiovasc. Surg., 92: $691,705,1986$.

18 LANSMAN, S. L.; ERGIN, A.; GRIEPP, R. B. - Treatment of acute aortic arch dissection. Ann. Thorac. Surg., 55: 816-817, 1993.

19 MERRILL, W. H.; ACHUFF, S. C.; WHITE Jr., R. I.; ROSS, R. S.; GOTT, V. L. - Late false aneurysm following replacement of ascending aorta: the problem

of the Teflon graft in combination with a silk suture. Ann. Thorac. Surg., 39: 271-274, 1985.

MOREIRA, L. P. P.: STOLF, N. A. G.; VIANNA, C. B.; PÊGO-FERNANDES, P. M.; PEREIRA-BARRETO, A. C.; VERGINELLI, G.; JATENE, A. D. - Fatores de risco na cirurgia das dissecçōes da aorta ascendente e arco aórtico. Rev. Bras. Cir. Cardiovasc., 2: $121-128,1987$

PÊGO-FERNANDES, P. M.; STOLF, N. A. G.; BEYRUTI, R.; MOREIR, L. F. P.; MADY, C.; JATENE, A. D. Resultados da substituição da aorta ascendente e valva aórtica com r eimplante de artérias coronárias. Arq. Bras. Cardiol., 55: 361-365, 1990.

REUL Jr, G. R. - The role oif suture in complications in vascular surgery and their relationship to pseudoaneurysm formation. In: BERNHARD, V. M. \& TOWNE, J. B. (eds.) Complications in vascular surgery. New York, Grune \& Stratton, 1980. p. 632.

ROCCHINI, A. P.; WEESNER, K. M.; HEIDELBERGER, K.; KEREN, D.; BEHRENDT, D.; ROSENTHAL, A. Porcine xenograft valve failure in children: an immunologic response. Circulation, 64 (Parte 2): III62 - III171, 1981.

SEGUIN, J. R.; FAPIER, J. M.; COLSON, P.; CHAPTAL, P. A. - Fibrin sealant improves surgical results of type $A$ acute aortic dissections. Ann. Thorac. Surg., 52: 745-749, 1991.

SVENSON, L. G.; CRAWFORD, S.; HESS, K. R.; COSELLI, J. S.; SAFI, H. J. - Composite valve graft replacement of the proximal aorta: comparison of techniques in 348 patients. Ann. Thorac. Surg., 54: 427-439, 1992.

VRANDECIC, M. P.; GONTIJO FILHO, B.; FANTINI, F. A. - New anticalcificant tanning of biological tissues: initial comparative analysis. Cardiovasc. Surg. (No prelo).

27 WESTABY, S.; PARRY, A.; GIANNOPOULOS, N.; PILLAI, R. - Replacement of the thoracic aorta with collagen-impregnated woven Dacron grafts: early results. J. Thorac. Cardiovasc. Surg., 106: 427-433, 1993. 